\title{
Fermi energy shift in deposited metallic nanotubes: A Raman scattering study
}

\author{
Benjamin Hatting, ${ }^{1, *}$ Sebastian Heeg, ${ }^{1}$ Kenichi Ataka, ${ }^{1}$ Joachim Heberle, ${ }^{1}$ Frank Hennrich, ${ }^{2}$ Manfred M. Kappes, ${ }^{2}$ \\ Ralph Krupke, ${ }^{2}$ and Stephanie Reich ${ }^{1}$ \\ ${ }^{1}$ Institut für Experimentalphysik, Freie Universität Berlin, Berlin, Germany \\ ${ }^{2}$ Institut für Nanotechnologie, Karlsruhe Institute of Technology, Karlsruhe, Germany \\ (Received 9 January 2013; revised manuscript received 25 March 2013; published 30 April 2013; corrected 1 May 2013)
}

\begin{abstract}
The longitudinal optical phonon of metallic nanotubes shifts by $23 \mathrm{~cm}^{-1}$ to lower energies when the nanotubes are deposited from a solution onto a substrate. The linewidth increases by $13 \mathrm{~cm}^{-1}$. The changes are explained in terms of shifts in the Fermi energy that influence the Kohn anomaly in the longitudinal optical phonon branch in metallic nanotubes. Using in situ electrochemical Raman measurements we show that the Fermi energy is $0.16 \mathrm{eV}$ below its intrinsic value in metallic nanotubes in solution. Our results impact the application of Raman spectroscopy to distinguish between metallic and semiconducting tubes by examining the high-energy mode line shape.
\end{abstract}

DOI: 10.1103/PhysRevB.87.165442

PACS number(s): 61.48.De, 63.22.Gh, 78.30.Na

The high-energy modes around $1600 \mathrm{~cm}^{-1}$ (also called $\mathrm{G}$ modes) are a prominent feature of single-walled carbon nanotube (SWNT) Raman spectra. They comprise out-ofphase in-plane vibrations tangential (TO) and parallel (LO) to the nanotube axis. The exact frequency of the LO and TO vibrations depends sensitively on the metallic or semiconducting character of the tubes. ${ }^{1-4}$ In semiconducting tubes, the TO mode is located at lower frequencies than the LO mode because of the nanotube curvature which softens the bonds along the circumference. ${ }^{5}$ In metallic tubes the LO phonon is broadened and downshifted by the interaction of the phonon with low-energy electron-hole pairs that results in a Kohn anomaly. ${ }^{6-8}$ The appearance of a broad peak at $1550 \mathrm{~cm}^{-1}$ is generally taken as indicative of the presence of metallic tubes. Recent studies claimed the absence of such an LO peak in armchair metallic tubes, ${ }^{9,10}$ despite earlier reports to the contrary. ${ }^{11,12}$ The study by Haroz et al. ${ }^{9}$ stresses the advantage of ensemble measurements in solution over measurements on individual tubes on a substrate. It was argued that ensemble studies are less sensitive to variations on the single-tube level and changes of the local environment. The argument implicitly assumes that the Raman spectra of tubes in solution remain unchanged upon deposition. This is particularly important since nanotube chirality enrichment and selection is done on tubes in solution, whereas nanotube devices such as field effect transistors are constructed on substrates.

In this paper, we show that the Raman line shape of nanotubes in solution and after deposition on a substrate differs strongly in metallic tubes. We observe an upshift and narrowing of the metallic LO peak in solution compared to on silicon, while the HEM of semiconducting tubes remain unchanged. The drastic changes are caused by Fermi energy shifts, lifting the Kohn anomaly. The shift amounts to $23 \mathrm{~cm}^{-1}$ and leads to hole doping as we demonstrate by combined electrochemical Raman measurements.

The sample investigated in this study is an enriched sample produced using size exclusion chromatography by gel filtration (Fraction 7 of Ref. 1; photoluminescence excitation map shown in Fig. S1 in Supplemental Material ${ }^{36}$ ) with the starting nanotube material produced by pulsed laser vaporation (PLV). The semiconducting species are enriched in the $(14,1)$, etc., branch; the sample has a narrow diameter distribution centered at $1.1 \mathrm{~nm}$. The tubes are suspended in aqueous solution with sodium cholate and a residual amount of sodium dodecyl sulfate as surfactants. The sample on silicon was prepared by depositing a single drop on a $\mathrm{Si} / \mathrm{SiO}_{2}$ wafer with a $300 \mathrm{~nm}$ oxide thickness and letting it dry in air.

Raman spectra were acquired with Dilor XY and XploRa spectrometers at wavelengths of 488, 633, 638, and $647 \mathrm{~nm}$. Spectra in solution and on substrates were recorded in microRaman backscattering configurations with $10 \times$ magnification objectives. The Dilor spectrometer uses a triple-grating subtractive monochromator and the XploRa features an edge filter to block Rayleigh-scattered light. Frequencies were calibrated using a neon lamp.

The laser spot illuminates ensembles of nanotubes in both the solution-based and the substrate supported samples. The spectra reflect the average Raman response of many different nanotubes. We verified the homogeneity of the deposited sample by taking multiple spectra at various positions.

Electrochemical measurements were performed using a 1:2.4 weight ratio mixture of $\mathrm{LiCl}_{4}$ in poly(ethylene oxide) (PEO; molecular weight 400) as the electrolyte. The chemicals were mixed as-received from Aldrich in air. The nanotube sample was deposited on a mica slide covered with a $100 \mathrm{~nm}$ $\mathrm{Au}$ layer and was used as the working electrode. $\mathrm{An} \mathrm{Ag} / \mathrm{AgCl}$ reference electrode (World Precision Instruments) and a silver wire acting as a counter electrode complete the electrochemical cell. We applied a gate voltage using a homemade voltage source that also monitored the current between the working and counter electrodes. This current did not exceed $2 \mu \mathrm{A}$ at gate voltages within a range of $\pm 1.4 \mathrm{~V}$. Use of the polymer electrolyte is necessary because aqueous electrolytes are only stable within a $1.3 \mathrm{~V}$ range. Outside this limited range, bubbles form in the electrolyte, rendering further measurements impossible.

Figure 1(a) shows HEM Raman spectra of ensembles of nanotubes in solution (blue trace) and after deposition on a silicon substrate (black trace) acquired with $647 \mathrm{~nm}$ excitation. The spectrum in solution shows a higher frequency peak at $1589 \mathrm{~cm}^{-1}$ and a shoulder at $1577 \mathrm{~cm}^{-1}$ with a full width at half maximum of $29 \mathrm{~cm}^{-1}$. One is tempted to assign the spectrum to semiconducting tubes with the typical dominating peak around $1590 \mathrm{~cm}^{-1}$. The spectrum measured under the 


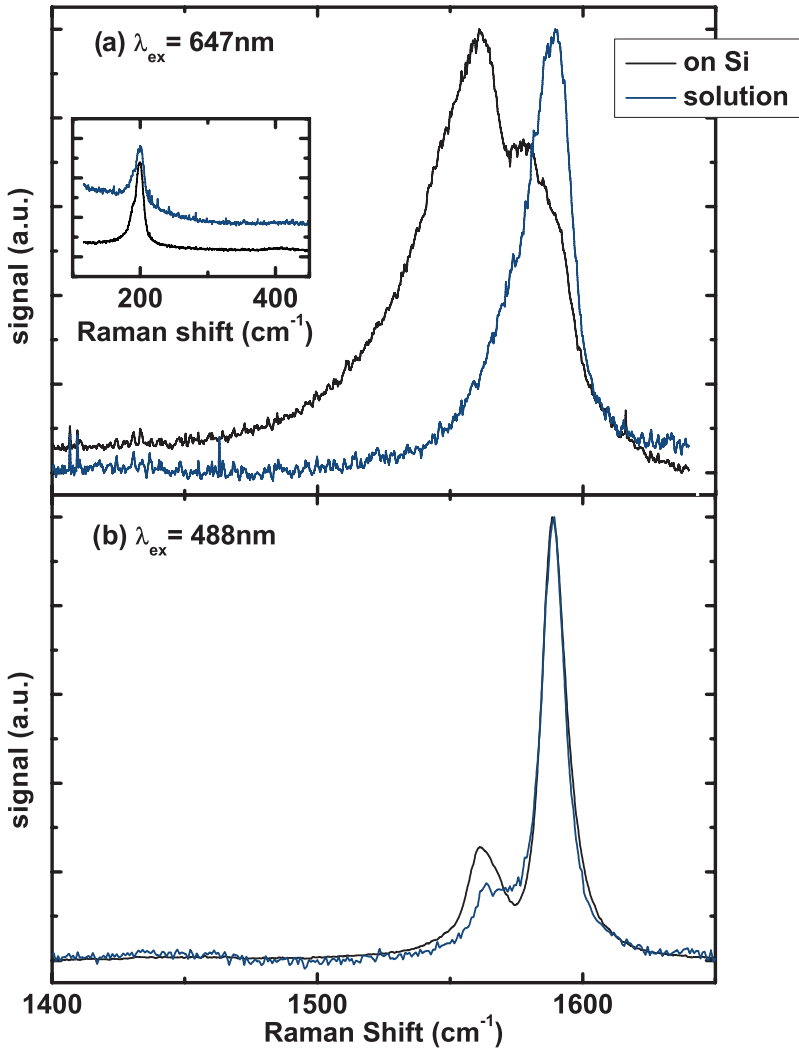

FIG. 1. (Color online) HEM Raman spectra recorded on ensembles of nanotubes in solution (blue trace) and on silicon (black trace) at wavelengths (a) 647 and (b) $488 \mathrm{~nm}$. The spectra at $647 \mathrm{~nm}$ exhibit drastic differences as discussed in the text, while the spectra at $488 \mathrm{~nm}$ are largely unchanged. The inset shows the RBM spectra at $647 \mathrm{~nm}$, which are very similar in solution and on silicon.

same conditions on silicon, in contrast, is dominated by a broad and asymmetric low-frequency component at $1554 \mathrm{~cm}^{-1}$ with a full width at half maximum of $42 \mathrm{~cm}^{-1}$ (see Fig. S3 in Supplemental Material $^{36}$ for a fit). This peak is clearly due to metallic LO vibrations.

A possible explanation for the change from semiconducting to metallic line shapes upon deposition is a change in the optical transition energies. However, the RBM spectra in the two experiments are almost identical [see inset of Fig. 1(a)], verifying constant resonance conditions. Due to the narrow resonance windows of RBMs even tiny changes in the optical transition energies result in drastic changes of the relative RBM intensities in ensemble measurements. ${ }^{13}$ The RBM data also allow identifying the tubes as the metallic $(15,0),(14,2),(13,4)$, and $(12,6)$ chiralites $(2 n+m=30$ branch $) .{ }^{13,14}$

For blue excitation the comparison between the two Raman spectra is qualitatively different. Here, the signal arises from semiconducting tubes and there are negligible differences between the spectra in solution and on silicon, as seen in Fig. 1(b). We also examined the HEM spectra of a HiPCO sample (not shown) and observed similar downshifts and narrowing of the metallic LO peak upon deposition. The effects were of smaller magnitude and more difficult to detect. Furthermore, the 2D Raman line of the enriched sample shifts by $10 \mathrm{~cm}^{-1}$ to smaller energies when depositing tubes on $\mathrm{Si}$, in analogy to the HEM behavior. (a)

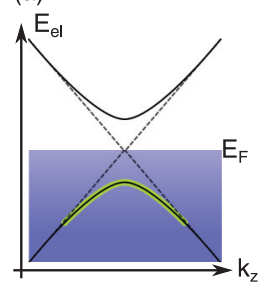

(d)

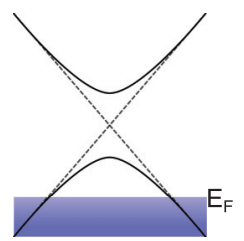

(g)

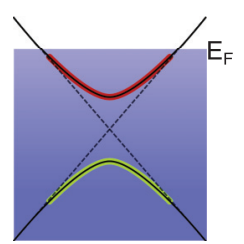

(b)

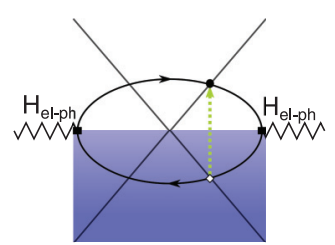

(e)

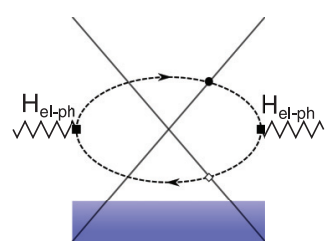

(h)

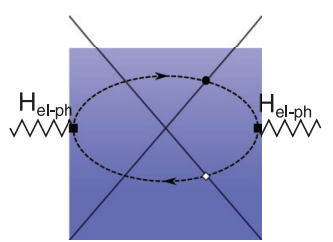

(c)

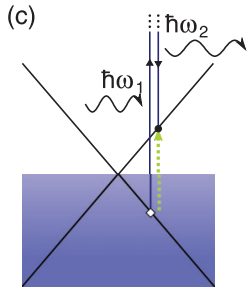

(f)

(i)

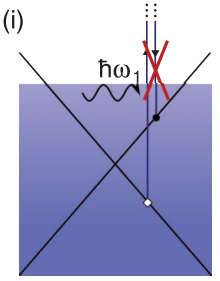

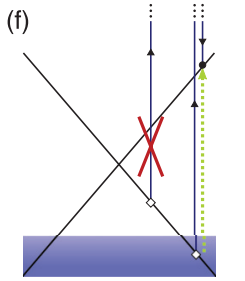

FIG. 2. (Color online) Effects of the Fermi energy on the HEM line shape of metallic SWNTs at intrinsic Fermi energy (top row) and Fermi energies below (middle row) and above (bottom row) the band gap. The effects of a dynamic band gap (solid black lines; intrinsic band structure shown as gray dashed lines) on the energy of the electronic system is shown in (a), (d), and (g). Occupied states that are moved to lower (higher) energies are highlighted in green (red). (b), (e), and (h) show the Feynman diagrams for the phonon self-energy due to the electron phonon interaction. This self-energy includes the resonant decay of the phonon into a zero-wave-vector electron-hole pair at intrinsic Fermi energies (green dashed line). At nonintrinsic Fermi energies the decay channel is blocked. The electronic Raman process that competes with the LO pathway and leads to the asymmetric line shape is shown in (c), (f), and (i). The Raman processes are also blocked at nonintrinsic Fermi energies.

The changes in the HEM spectra of metallic tubes in Fig. 1(a) are due to shifts in the Fermi energy upon deposition. The Fermi energy affects the metallic LO peak in three ways as summarized in Fig. 2. The metallic LO frequency is lower than in semiconducting tubes because of the Kohn anomaly at $\mathbf{q}=0$ ( $\Gamma$ point $).{ }^{15,16}$ In a quasistatic picture the LO displacement leads to the opening of a periodic gap in a nanotube. ${ }^{17}$ This band gap reduces the time-averaged total electronic energy, Fig. 2(a). Less energy is required to excite the LO mode, resulting in a phonon softening. The Kohn anomaly was described more rigorously in DFT calculations $^{5,7}$ and in calculations of the phonon self-energy due to the electron-phonon interaction. ${ }^{6,18}$ Second, the width of the metallic LO peak is affected by the Fermi energy. Under intrinsic conditions the LO phonon can decay into a low-energy zero-wave-vector electron-hole pair in the two bands crossing at the Fermi energy, ${ }^{19}$ as shown as a green dashed arrow in Fig. 2(b). This relaxation channel vanishes with a shift in Fermi energy. Third, the metallic LO peak at intrinsic Fermi energy exhibits an asymmetric line shape and is fitted with Fano profiles. This is due to quantum interference between the discrete LO phonon and the continuous electronic 
Raman scattering process; ${ }^{20}$ the latter process is shown in Fig. 2(c). ${ }^{21,22}$ All three mechanisms depend sensitively on the position of the Fermi energy. They are blocked or not in effect when the Fermi energy is not at its intrinsic value (see second and third row in Fig. 2).

We assign the lower frequency peaks of Fig. 1(a) in solution and on silicon to metallic LO phonons. The LO in solution is higher frequency, narrower, and more symmetric because the Fermi energy is shifted from its intrinsic value. The downshifted, broad, and asymmetric metallic LO peak on silicon is characteristic of metallic tubes with intrinsic Fermi energies. Quantitatively, the metallic LO shift has a magnitude of $23 \mathrm{~cm}^{-1}$ and the full width at half maximum changes by $13 \mathrm{~cm}^{-1}$.

To confirm our interpretation and quantify the position of the Fermi energy we performed in situ electrochemical Raman measurements. ${ }^{12,23,24}$ HEM Raman spectra recorded with gate voltages ranging from -1.4 to $0 \mathrm{~V}$ are shown in Fig. 3(a). Negative gate voltages correspond to negative Fermi energy shifts, that is, hole doping. Spectra acquired at positive gate voltages (electron doping) show similar behavior. The most obvious development in Fig. 3(a) is an upshift and a narrowing of the lowest frequency metallic LO peak with increasing $\left|V_{g}\right|$. There is excellent agreement between the line shape of the electrochemical spectrum at $-0.5 \mathrm{~V}$ and the spectrum recorded in solution; see Fig. 3(b). We reproduce the spectrum in solution by deliberately shifting the Fermi energy, confirming our interpretation.

We now use the broadening of the metallic LO peak with gate voltage to calibrate the position of the Femi energy. Figure 4(a) compares the experimentally observed peak width as a function of gate voltage with the theoretical dependence of the peak width on Fermi energy. The broadening decreases to

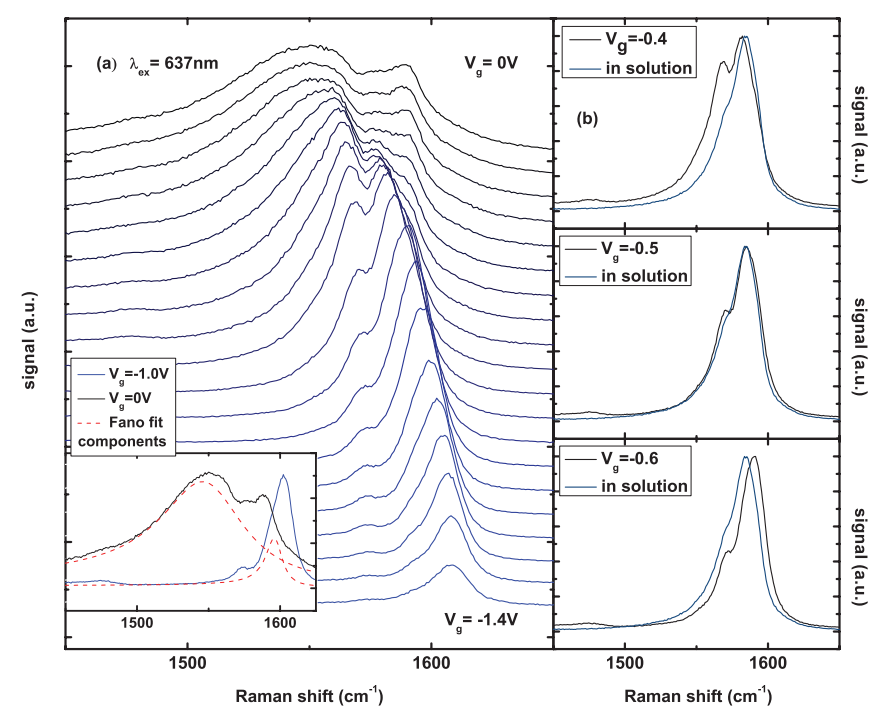

FIG. 3. (Color online) HEM Raman spectra acquired at $638 \mathrm{~nm}$ under application of electrochemical gating. A gate voltage sweep from $-1.4 \mathrm{~V}$ to $0 \mathrm{~V}$ is shown in (a). The inset shows two examples of the Fano fit component for gate voltages of $0 \mathrm{~V}$ and $-1.0 \mathrm{~V}$. The spectra acquired at gate voltages of $-0.4,-0.5$, and $-0.6 \mathrm{~V}$ are each compared to the spectrum in solution in (b). The line shape matches at a gate voltage of $-0.5 \mathrm{~V}$.
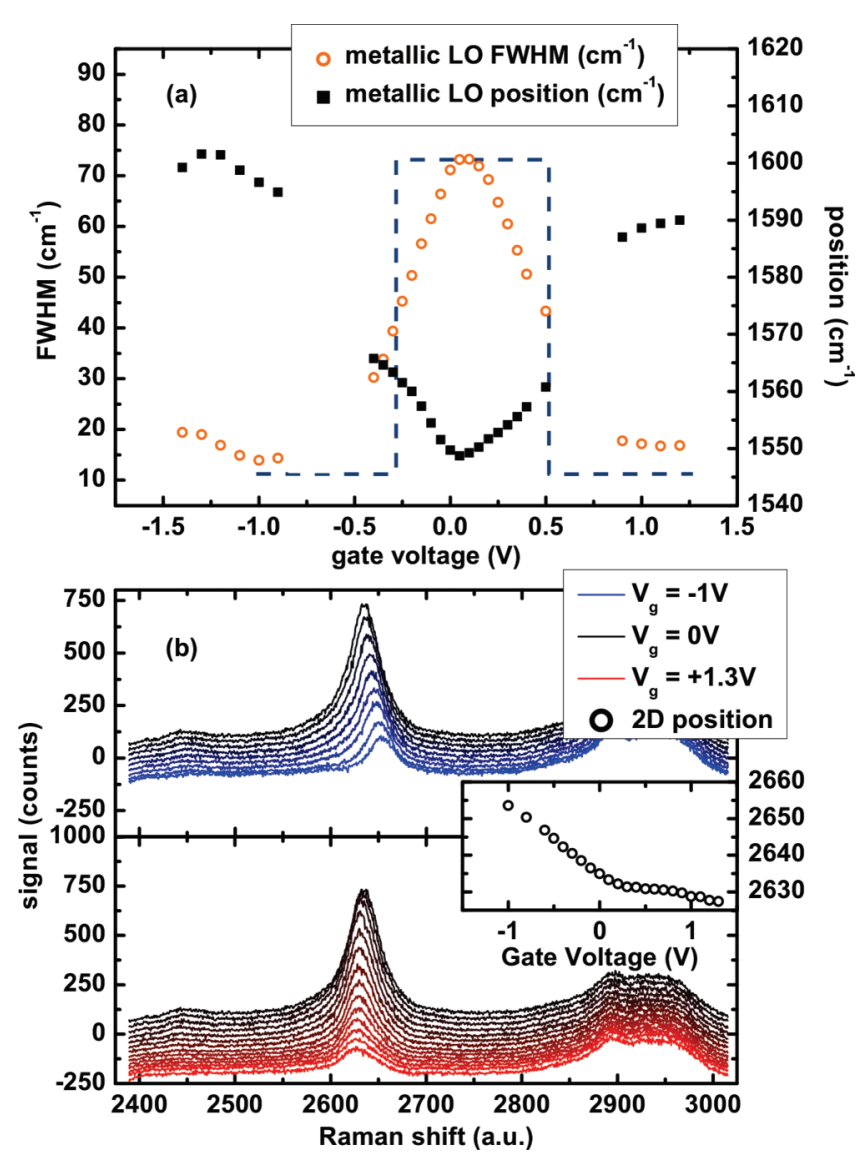

FIG. 4. (Color online) (a) Linewidth (left axis) and peak position (right axis) of the metallic LO peak as a function of gate voltage together with the theoretical prediction for the phonon linewidth at $0 \mathrm{~K}$ without electronic broadening. The intersections at half of the maximum broadening occur at Fermi energy shifts of $\pm \hbar \omega_{p h} / 2$. (b) 2D Raman spectra acquired at negative (top panel) and positive (bottom panel) gate voltages. The spectra at negative voltages exhibit a progressive upshift, while the spectra at positive voltages exhibit a downshift of smaller magnitude. The $2 \mathrm{D}$ peak position as a function of gate voltage is plotted in the inset.

half of its maximum value at Fermi energies of plus and minus half the phonon energy, that is, $\pm 0.1 \mathrm{eV}^{6,25}$ Experimentally the broadening is reduced to half of its maximum at -0.25 and $+0.5 \mathrm{~V}$; see Fig. 4(a). The rate of change of the Fermi energy with the gate voltage, or gating efficiency, is $0.2 \mathrm{eV}$ (the phonon energy) per $0.75 \mathrm{~V}$ (voltage difference between crossing points) or $0.27 \mathrm{eV} / \mathrm{V}$, comparable to $0.4-0.7 \mathrm{eV} / \mathrm{V}$ reported earlier. ${ }^{26,27}$ The spectrum in solution, middle panel in Fig. 4(b), corresponds to a Fermi energy shift $\left|\Delta E_{F}\right|=$ $(0.16 \pm 0.03) \mathrm{eV}$.

Next we determine the direction of the shift in Fermi energy by examining the $2 \mathrm{D}$ mode under electrochemical gating. Figure 4(b) shows the spectral development under negative (upper panel) and positive (lower panel) gate voltages. For negative gate voltages, the peak exhibits an upshift, whereas it exhibits a downshift by a smaller absolute magnitude for positive gate voltages. This asymmetry of the $2 \mathrm{D}$ mode with the sign of the Fermi energy shift is due to changes in the resonance conditions; it allows us to determine the direction 
of the Fermi energy shift. As the 2D peak in solution is higher in frequency than on a substrate, the Fermi energy in solution is below its intrinsic value, corresponding to hole doping. The position of the Fermi energy in solution is therefore $\Delta E_{F}=-(0.16 \pm 0.03) \mathrm{eV}$ below the band crossing point. The spectrum recorded on silicon exhibits a metallic LO peak. Nevertheless following the same arguments as for tubes in solution electrochemical measurements reveal $\Delta E_{F}=-(0.07 \pm 0.02) \mathrm{eV}$.

We now discuss possible explanations for the positions of the Fermi level. Strano et al. ${ }^{28}$ investigated the effects of changing the $\mathrm{pH}$ value of a SWNT solution on the HEM line shape. They found that metallic tubes protonate at $\mathrm{pH}$ around 5 , which leads to a withdrawal of electrons. They observed an upshift of the metallic LO peak after protonation. Strano et al. ${ }^{28}$ also demonstrated that the surfactant shifts the $\mathrm{pH}$ value at which tubes protonate to more neutral values. A slightly acidic $\mathrm{pH}$ in our solution would thus explain the Fermi energy shift we observe in our sample.

After deposition, the Fermi energy continues to be below the intrinsic value. $p$-type behavior was observed in nanotube and graphene field-effect transistors on $\mathrm{Si}$ under ambient conditions in many studies ${ }^{29-32}$ and attributed to ambient oxygen. This agrees with our results. Gaur and Shim $^{33}$ found a narrowing and upshift of the LO peak in metallic nanotubes under ambient conditions compared to after argon annealing. They ascribed the behavior to nonintrinsic positions of the Fermi energy under ambient conditions due to oxygen adsorption - in agreement with us - but failed to determine the sign and magnitude of the energy shift.

In summary, we have conclusively demonstrated two Fermi energy shifts in metallic nanotubes: away from the intrinsic value in solution and back towards its intrinsic value after deposition on silicon. The shifts manifest themselves in a changing HEM line shape of metallic tubes, with an upshift and narrowing of the metallic LO peak in solution. Electrochemistry allowed us to quantitatively determine the sign and magnitude of the observed Fermi energy shifts in solution $(-0.16 \mathrm{eV})$ and on silicon $(-0.07 \mathrm{eV})$. Our results have implications for the application of Raman spectroscopy to estimate the fraction of metallic and semiconducting tubes in bulk nanotube samples because Fermi energy shifts might lead to the underestimation of the fraction of metallic tubes. Spectra are preferably acquired on silicon where the Fermi energy is closer to its intrinsic value and the metallic LO exhibits the characteristic downshift, broadening, and asymmetry. This is particularly recommendable for volume samples intended for electronic applications where the dominance of either semiconducting or metallic species is critical. ${ }^{34,35}$

S.H. and S.R. acknowledge funding by the ERC (Grant No. 210642). *benjamin.hatting@fu-berlin.de

${ }^{1}$ C. Blum, N. Stürzl, F. Hennrich, S. Lebedkin, S. Heeg, H. Dumlich,

S. Reich, and M. M. Kappes, ACS Nano 5, 2847 (2011).

${ }^{2}$ D. Chattopadhyay, I. Galeska, and F. Papadimitrakopoulos, J. Am. Chem. Soc. 125, 3370 (2003).

${ }^{3}$ Y. Maeda, S.-i. Kimura, M. Kanda, Y. Hirashima, T. Hasegawa, T. Wakahara, Y. Lian, T. Nakahodo, T. Tsuchiya, T. Akasaka, J. Lu, X. Zhang, Y. Yu, S. Nagase, S. Kazaoui, N. Minami, T. Shimizu, H. Tokumoto, and R. Saito, J. Am. Chem. Soc. 127, 10287 (2005).

${ }^{4}$ H. Li, B. Zhou, Y. Lin, L. Gu, W. Wang, K. A. S. Fernando, S. Kumar, L. F. Allard, and Y.-P. Sun, J. Am. Chem. Soc. 126, 1014 (2004).

${ }^{5}$ S. Piscanec, M. Lazzeri, J. Robertson, A. C. Ferrari, and F. Mauri, Phys. Rev. B 75, 035427 (2007).

${ }^{6}$ K. Ishikawa and T. Ando, J. Phys. Soc. Jpn. 75, 084713 (2006).

${ }^{7}$ N. Caudal, A. M. Saitta, M. Lazzeri, and F. Mauri, Phys. Rev. B 75, 115423 (2007).

${ }^{8}$ J. C. Tsang, M. Freitag, V. Perebeinos, J. Liu, and P. Avouris, Nat. Nanotechnol. 2, 725 (2007).

${ }^{9}$ E. H. Haroz, J. G. Duque, W. D. Rice, C. G. Densmore, J. Kono, and S. K. Doorn, Phys. Rev. B 84, 121403 (2011).

${ }^{10}$ M. Paillet, T. Michel, A. Zahab, D. Nakabayashi, V. Jourdain, R. Parret, J. Meyer, and J. L. Sauvajol, Phys. Status Solidi B 247, 2762 (2010).

${ }^{11}$ K. I. Sasaki, R. Saito, G. Dresselhaus, M. S. Dresselhaus, H. Farhat, and J. Kong, Phys. Rev. B 77, 245441 (2008).

${ }^{12}$ J. S. Park, K. Sasaki, R. Saito, W. Izumida, M. Kalbac, H. Farhat, G. Dresselhaus, and M. S. Dresselhaus, Phys. Rev. B 80, 081402 (2009).
${ }^{13}$ J. Maultzsch, H. Telg, S. Reich, and C. Thomsen, Phys. Rev. B 72, 205438 (2005)

${ }^{14}$ H. Telg, J. Maultzsch, S. Reich, F. Hennrich, and C. Thomsen, Phys. Rev. Lett. 93, 177401 (2004).

${ }^{15}$ W. Kohn, Phys. Rev. Lett. 2,393 (1959).

${ }^{16}$ S. Piscanec, M. Lazzeri, F. Mauri, A. C. Ferrari, and J. Robertson, Phys. Rev. Lett. 93, 185503 (2004).

${ }^{17}$ O. Dubay, G. Kresse, and H. Kuzmany, Phys. Rev. Lett. 88, 235506 (2002).

${ }^{18}$ T. Ando, J. Phys. Soc. Jpn. 75, 124701 (2006).

${ }^{19}$ Y. Wu, J. Maultzsch, E. Knoesel, B. Chandra, M. Y. Huang, M. Y. Sfeir, L. E. Brus, J. Hone, and T. F. Heinz, Phys. Rev. Lett. 99, 027402 (2007)

${ }^{20}$ M. V. Klein, in Light Scattering in Solids I: Introductory Concepts, Topics in Applied Physics, Vol. 8, edited by M. Cardona (SpringerVerlag, Berlin, 1983), p. 147.

${ }^{21}$ U. Fano, Phys. Rev. 124, 1866 (1961).

${ }^{22}$ F. Cerdeira, T. A. Fjeldly, and M. Cardona, Phys. Rev. B 8, 4734 (1973).

${ }^{23}$ K. T. Nguyen, A. Gaur, and M. Shim, Phys. Rev. Lett. 98, 145504 (2007).

${ }^{24}$ M. Kalbac, L. Kavan, L. Dunsch, and M. S. Dresselhaus, Nano Lett. 8, 1257 (2008)

${ }^{25}$ T. Ando, J. Phys. Soc. Jpn. 77, 014707 (2008).

${ }^{26}$ S. B. Cronin, R. Barnett, M. Tinkham, S. G. Chou, O. Rabin, M. S. Dresselhaus, A. K. Swan, M. S. Unlu, and B. B. Goldberg, Appl. Phys. Lett. 84, 2052 (2004).

${ }^{27}$ P. M. Rafailov, J. Maultzsch, C. Thomsen, and H. Kataura, Phys. Rev. B 72, 045411 (2005). 
${ }^{28}$ M. S. Strano, C. B. Huffman, V. C. Moore, M. J. O'Connell, E. H. Haroz, J. Hubbard, M. Miller, K. Rialon, C. Kittrell, S. Ramesh, R. H. Hauge, and R. E. Smalley, J. Phys. Chem. B 107, 6979 (2003).

${ }^{29}$ V. Derycke, R. Martel, J. Appenzeller, and P. Avouris, Appl. Phys. Lett. 80, 2773 (2002).

${ }^{30}$ S.-H. Hur, M.-H. Yoon, A. Gaur, M. Shim, A. Facchetti, T. J. Marks, and J. A. Rogers, J. Am. Chem. Soc. 127, 13808 (2005).

${ }^{31}$ C. M. Aguirre, P. L. Levesque, M. Paillet, F. Lapointe, B. C. St-Antoine, P. Desjardins, and R. Martel, Adv. Mater. 21, 3087 (2009).
${ }^{32}$ P. L. Levesque, S. S. Sabri, C. M. Aguirre, J. Guillemette, M. Siaj, P. Desjardins, T. Szkopek, and R. Martel, Nano Lett. 11, 132 (2010).

${ }^{33}$ A. Gaur and M. Shim, Phys. Rev. B 78, 125422 (2008).

${ }^{34}$ P. G. Collins, M. S. Arnold, and P. Avouris, Science 292, 706 (2001).

${ }^{35}$ R. Krupke, F. Hennrich, H. von Lohneysen, and M. M. Kappes, Science 301, 344 (2003).

${ }^{36}$ See Supplemental Material at http://link.aps.org/supplemental/ 10.1103/PhysRevB.87.165442 for additional figures. 